Faculty of Biology, Medicine and Health, University of Manchester, Manchester, UK

2 Centre for Primary Care and Health Services Research, Division of Population Health, Health Services Research and Primary Care, School of Health Sciences, Faculty of Biology, Medicine and Health, University of Manchester, Manchester, UK

Correspondence to: M Sperrin matthew.sperrin@manchester.ac.uk Cite this as: BMJ 2020;371:m3777 http://dx.doi.org/10.1136/bmj.m3777 Published: 21 October 2020

\title{
Prediction models for covid-19 outcomes
}

\section{Reasons to be cautious \\ Matthew Sperrin, ${ }^{1}$ Brian McMillan ${ }^{2}$}

Robust models that predict the prognosis of coronavirus 2019 (covid-19) are urgently needed to support decisions about shielding, hospital admission, treatment, and population level interventions. With cases increasing in the UK and elsewhere, and winter approaching, such models could have a rapid clinical impact. Two linked articles report on two newly developed covid-19 prediction models. QCOVID is a risk prediction model for covid-19 related mortality for use in the general population (doi:10.1136/bmj.m3731), ${ }^{1}$ whereas the $4 \mathrm{C}$ mortality score is for use on admission to hospital (doi:10.1136/bmj.m3339). ${ }^{2}$ Notably, these models are of higher quality than others published to date, ${ }^{3}$ having been developed using ample sample sizes, ${ }^{4}$ with generally appropriate modelling choices, and suitably internally validated and reported. ${ }^{6} 6$ Nevertheless, we sound a note of caution in their use. QCOVID predicts the risk of catching and dying from (or being admitted to hospital with) covid-19 in the general population. ${ }^{1}$ The authors rightly emphasise the fact that predicting separately either the probability of catching covid-19 or the probability of dying from it is not possible, owing primarily to incomplete knowledge of who actually has the disease. However, this conflation causes limitations in the model's application. The risk of catching covid-19 depends on an individual's behaviour and the local dynamics of the disease, which are not modelled by QCOVID. These dynamics, such as local disease prevalence, change rapidly. Therefore, calibration of the model is likely to deteriorate rapidly. Moreover, recent data show a shift in the age distribution of cases towards younger people; discrimination of the model may also drop, therefore, as age is a strong predictor. QCOVID is, however, described as a "living" model ${ }^{1}$; with regular updating, these problems can be mitigated. ${ }^{7}$

A further challenge is that the predictions made reflect interventions in place at the time the model was developed. A "low risk" prediction generated by the model might reflect active steps taken by similar people in the past to lower their risk, such as shielding. Therefore, using a low risk prediction to support a decision to return to work, for example, is problematic. Explicit separation of baseline risk factors and interventions can, in principle, be achieved through issuing counterfactual predictions, which provide a risk estimate assuming certain interventions. $^{89}$ Of particular interest for decision making is the counterfactual prediction generated when no preventive measures (such as shielding) are taken. ${ }^{10}$

QCOVID might, with these caveats, be used to inform national guidelines on shielding and employment legislation regarding who can reasonably be required to remain in, or return to, specific work environments. Risk estimates from QCOVID could also inform discussions between clinicians and patients. If challenge trials of covid -19 treatments are to go ahead, ${ }^{11}$ QCOVID could help scientists to target recruitment and enable potential participants to make informed decisions about their risks from taking part. Should effective vaccines be developed, it could inform decisions about which groups should be prioritised.

The ${ }_{4} \mathrm{C}$ mortality score, calculated at hospital admission, predicts in-hospital mortality among patients with confirmed or likely covid-19. ${ }^{2}$ Here, the authors explicitly suggest that the model should be used for decision support, noting, for example, that "patients with a $4 \mathrm{C}$ mortality score falling within the low risk groups (mortality rate $1 \%$ ) might be suitable for management in the community." This does not account for the "treatment paradox": these patients may seem to be at low risk because of the interventions that similar patients in the development cohort received in hospital. ${ }^{12}$ Again, counterfactual prediction modelling offers a potential solution. ${ }^{9}$ Furthermore, with patient management, and potentially the disease itself, changing rapidly, the ${ }_{4} \mathrm{C}$ model must also be updated regularly. ${ }^{7}$

Some clinical scenarios exist in which these risk calculators are of limited value. Neither model would help community based clinicians to determine whether patients being video triaged should be seen in person or admitted to hospital. Greenhalgh et al have developed guiding principles, 13 but much remains to be done. Future studies could assess the clinical and cost benefits of supplying patients at high risk with equipment to record their vital signs from home (pulse oximeters, blood pressure monitors, thermometers, and peak expiratory flow rate meters), as this could improve the discrimination of any risk assessment tools based on remote triage.

To conclude, the $4 \mathrm{C}$ and QCOVID models are likely to be helpful and represent a step forward in the quality of prognosis models for covid-19. Given the rapidly changing nature of the disease and its management, we emphasise the need to update these models regularly and monitor their performance closely over time and space. Care must also be taken when interpreting the predictions generated by these models: they reflect the risk for a patient taking similar measures, and receiving similar care, to similar patients in the past, not the risk to a patient if no actions are taken. Improved data on incident cases of covid-19 will allow greater granularity in prediction. With these caveats, we support the 


\section{continued validation and impact assessment of these models.}

Competing interests: The BMJ has judged that there are no disqualifying financial ties to commercial companies. The authors declare no other interests."

Provenance and peer review: Commissioned; not peer reviewed.

1 Clift AK, Coupland CAC, Keogh RH, etal. Living risk prediction algorithm (QCOVID) for risk of hospit admission and mortality from coronavirus-19 in adults: national derivation and validation cohort study. BMJ2020;371:m3731.

2 Knight SR, Ho A, Pius R, etallSARIC4C investigators. Risk stratification of patients admitted to hospital with covid-19 using the ISARIC WHO Clinical Characterisation Protocol: development and validation of the 4C Mortality Score. BMJ 2020;370:m3339. doi: 10.1136/bmj.m3339 pmid: 32907855

3 Wynants L, Van Calster B, Bonten MM, etal. Prediction models for diagnosis and prognosis of covid-19 infection: systematic review and critical appraisal. BMJ2020;369:m1328. doi: 10.1136/bmj.m1328 pmid: 32265220

4 Riley RD, Ensor J, Snell KIE, etal. Calculating the sample size required for developing a clinical prediction model. BMJ2020;368:m441. doi: 10.1136/bmj.m441 pmid: 32188600

5 Steyerberg EW, Vickers AJ, Cook NR, etal. Assessing the performance of prediction models: a framework for traditional and novel measures. Epidemiology 2010;21:128-38. doi: 10.1097/EDE.0b013e3181c30fb2 pmid: 20010215

6 Collins GS, Reitsma JB, Altman DG, Moons KG. Transparent Reporting of a multivariable prediction model for Individual Prognosis or Diagnosis (TRIPOD): the TRIPOD statement. Ann Intern Med 2015;162:55-63. doi: 10.7326/M14-0697 pmid: 25560714

7 Jenkins DA, Sperrin M, Martin GP, Peek N. Dynamic models to predict health outcomes: current status and methodological challenges. Diagn Progn Res 2018;2:23. doi: 10.1186/s41512-018-0045-2 pmid: 31093570

8 van Geloven N, Swanson SA, Ramspek CL, etal. Prediction meets causal inference: the role of treatment in clinical prediction models. Eur J Epidemiol 2020;35:619-30. doi: 10.1007/s10654-020-00636-1 pmid: 32445007

9 Dickerman BA, Hernán MA. Counterfactual prediction is not only for causal inference. Eur J Epidemiol 2020;35:615-7. doi: 10.1007/s10654-020-00659-8 pmid: 32623620

10 Sperrin M, Martin GP, Pate A, Van Staa T, Peek N, Buchan I. Using marginal structural models to adjust for treatment drop-in when developing clinical prediction models. Stat Med 2018;37:4142-54. doi: 10.1002/sim.7913 pmid: 30073700

11 World Health Organization. Key criteria for the ethical acceptability of COVID-19 human challenge studies. 2020. https://apps.who.int/iris/bitstream/handle/10665/331976/WHO-2019-nCoVEthics_criteria-2020.1-eng.pdf?ua=1.

12 Cheong-See F, Allotey J, Marlin N, etal. Prediction models in obstetrics: understanding the treatment paradox and potential solutions to the threat it poses. BJOG 2016;123:1060-4. doi: 10.1111/1471-0528.13859 pmid: 26806638

This is an Open Access article distributed in accordance with the Creative Commons Attribution Non Commercial (CC BY-NC 4.0) license, which permits others to distribute, remix, adapt, build upon this work non-commercially, and license their derivative works on different terms, provided the original work is properly cited and the use is non-commercial. See: http://creativecommons.org/licenses/by$\mathrm{nc} / 4.0 /$. 